\title{
The Role of External Acidity of Meso-/microporous Zeolites in Determining Selectivity for Acid-catalyzed Reactions of Benzyl alcohol
}

Laleh Emdadi ${ }^{1}$, Su Cheun $\mathrm{Oh}^{1}$, Yiqing Wu ${ }^{1}$, Shirin Norooz Oliaee ${ }^{2}$, Yuxia Diao ${ }^{3}$, Guanghui Zhu ${ }^{4}$ and Dongxia $\mathrm{Liu}^{1 *}$

1. Department of Chemical and Biomolecular Engineering, University of Maryland, College Park, MD, 20742

2. Department of Chemical and Biomolecular Engineering, University of Akron, Akron, OH, 44325

3. Research Institute of Petroleum Processing, SINOPEC, 18 Xueyuan Road, Beijing, China, 10083

4. School of Chemical and Biomolecular Engineering, Georgia Institute of Technology, Atlanta, Georgia, 30332

*Corresponding author:

Prof. Dongxia Liu

Email: liud@umd.edu

Phone: (+1) 301-405-3522

Fax: (+1) 301-405-0523 
Abstract: A comparison of selectivity in catalytic conversion of benzyl alcohol in mesitylene on hybrid lamellar-bulk MFI (HLBM) zeolite materials containing dual meso-/microporosity showed that the external Brønsted acidity in meso-/microporous MFI zeolites effectively impact on selectivity of the parallel alkylation and etherification reactions. HLBM zeolites, consisting of crystalline bulk microporous core and lamellar mesoporous shell, not only catalyzed the parallel reactions on the external environments (external surface and mesopore) but also etherification reaction in the internal environment (micropore) as illustrated by the completely suppressed alkylation and retained residual etherification reactions after 2,6-di-tert-butylpyridine (DTBP) poisoning. A systematic study of HLBM zeolites with tunable meso-/microporous domain sizes achieved by a dual template assisted synthesis revealed that parallel alkylation and etherification reactions are tailored by the tunable external surface area and external acidity of the HLBM zeolites. The external alkylation and etherification reaction rates as a function of cumulative DTBP addition suggested Brønsted acid sites with different strengths on external environments of the HLBM zeolites, which influenced the external etherification reaction, but not as significantly as the alkylation reaction. The evidence shown here for the involvement of external acidity in catalyzing parallel reactions and for the role of external acidity with variable strengths in HLBM zeolite materials extends the scope of observed catalytic behaviors of meso/microporous zeolite materials beyond those reflecting transport effects and accessibility of acid sites.

Keywords: Meso-/microporous zeolite; Hierarchical materials; Selectivity; Mesitylene; Benzyl alcohol; Dual template synthesis; Acidity; Acid strength. 


\section{Introduction}

The meso-/microporous zeolites couple the catalytic features of microporosity and the improved mass transport consequence of mesoporosity in a single material, possessing the capacity of overcoming the mass-transfer constraints and the resultant low catalyst utilization, a major drawback in most reactions catalyzed by the conventional microporous zeolites.[1-6] A variety of zeolites containing structured porosity on micro- and mesopore levels have been created in the past decade[2, 7-12] and the catalytic tests showed that the meso-/microporous zeolites have higher reaction rates and/or different selectivity in comparison with their microporous zeolite analogues which consist exclusively of the microporosity. The enhanced catalytic reaction rate and/or altered selectivity on meso-/microporous zeolites has been attributed to the enhanced accessibility to active sites and transport characteristics of these materials[13-16], while the role of external acidity (active sites on external surface and in pore mouth region) and systematically tailoring selectivity of external acid sites in the meso/microporous zeolite materials have remained rarely accessible.

Recently, we reported a simple one-step synthesis of the hybrid lamellar-bulk MFI (HLBM) zeolite with tunable meso-/microporous domains by a mixed template system (dual templates).[17, 18] The crystalline bulk zeolite core and lamellar zeolite shell were formed simultaneously in the hydrothermal crystallization step with the assistance of dual templates, tetrapropyl ammonium hydroxide $(\mathrm{TPAOH})$ and diquaternary ammonium surfactant $\left(\left[\mathrm{C}_{22} \mathrm{H}_{45^{-}}\right.\right.$ $\left.\left.\mathrm{N}^{+}\left(\mathrm{CH}_{3}\right)_{2}-\mathrm{C}_{6} \mathrm{H}_{12}-\mathrm{N}^{+}\left(\mathrm{CH}_{3}\right)_{2}-\mathrm{C}_{6} \mathrm{H}_{13}\right] \mathrm{Br}_{2},\left(\mathrm{C}_{22-6-6}\right)\right)$, respectively. By tuning the concentration of $\mathrm{C}_{22-}$ 6-6 and the synthesis conditions such as ageing temperature and time of the zeolite synthetic gel in the syntheses, the domain sizes of the lamellar shell and bulk core and thus the meso- and microporosity of the HLBM zeolite particles are simply modulated.[17] The capability of tuning 
the lamellar and bulk domain sizes of the HLBM zeolite creates the opportunity of designing materials with spatially controlled morphology that have potential advantages for various applications. For example, the similar type of hybrid zeolitic structure, prepared by epitaxial growth of a layered MFI zeolite on the surface of a pre-synthesized bulk MFI, showed enhanced permeability for $\mathrm{CO}_{2}$ and good $\mathrm{CO}_{2} / \mathrm{CH}_{4}$ selectivity for gas separations than bare bulk MFI zeolite when they were processed into HLBM zeolite/polyimide and bulk MFI zeolite/polyimide composite membranes.[19] This performance enhancement is due to the highly roughened surface morphology of HLBM zeolite that results in excellent adhesion to a dense glassy polymer. The chemical compositions, particularly acid sites associated with framework Al, could also be spatially modulated. The spatially modulated acid sites would influence the performance of zeolite materials, notably in the area of catalysis.

In this work, we aim to study the physicochemical properties and catalytic behaviors of HLBM zeolites prepared by one-step dual template assisted synthesis method. X-ray diffraction (XRD), scanning electron microscopy (SEM), transmission electron microscopy (TEM), nuclear magnetic resonance (NMR), argon (Ar) adsorption and Fourier transformation infrared (FTIR) spectroscopy of adsorbed pyridine were used to characterize the structural and acidity properties of HLBM zeolite materials. The spatial distribution of acid sites, either on external surface/pore mouth region or in micropores of HLBM zeolite, was identified by organic base titration method. The catalytic behavior of external acid sites in HLBM zeolite catalysts was tested using a parallel reaction, alkylation of mesitylene by benzyl alcohol to 1,3,5-trimethyl-2-benzylbenzene and selfetherification of benzyl alcohol to dibenzyl ether (scheme shown in S2 of the Supplementary Information), respectively. It is shown that fractions of external Brønsted acid sites were modulated when the domain sizes of the lamellar shell and bulk core were varied in HLBM 
zeolite. The spatially controlled acid site distribution effectively impacts on selectivity of the HLBM zeolite in catalyzing parallel chemical reactions. The study on parallel alkylation and etherification reactions over HLBM zeolite also indicated the potential of HLBM zeolite catalysts in catalyzing bulky molecules involved Friedel-Crafts alkylation/acylation and etherification/esterification reactions.

\section{Experimental}

2.1. Synthesis of HLBM zeolite catalysts. The method for synthesis of HLBM zeolite catalysts was similar to our previous work[17]. The HLBM zeolite was prepared by dissolving $0.0111 \mathrm{~g}$ sodium hydroxide $(\mathrm{NaOH}, \geq 97 \%$ purity) in $11.5 \mathrm{~g}$ deionized water (DI) followed by addition of $5.075 \mathrm{~g}$ tetrapropylammonium hydroxide (TPAOH, $40 \%(\mathrm{w} / \mathrm{w})$ ) to the solution. Then, $0.0567 \mathrm{~g}$ of aluminum isopropoxide was dissolved in the solution using sonication at room temperature for $0.5 \mathrm{~h}$. Afterwards, $5.78 \mathrm{~g}$ tetraethyl orthosiliccate (TEOS, 98\% purity) was added, and the resultant mixture was stirred vigorously at room temperature for $8 \mathrm{~h}$. The $\mathrm{C}_{22-6-6}$ solution that was prepared by dissolving the desired amount in $8.5 \mathrm{~g}$ of DI water at $333 \mathrm{~K}$ was added to above mixture. The resultant zeolite synthesis gel was continuously stirred at room temperature for $2 \mathrm{~h}$ before transferring it into a Teflon-lined stainless-steel autoclave. Finally, crystallization of zeolite was conducted for 5 days in the autoclaves tumbling vertically in an oven heated at $423 \mathrm{~K}$. After crystallization, the zeolite sample was filtered, washed with DI water, and dried at 343 K overnight.

The as-synthesized HLBM zeolite was then calcined at $873 \mathrm{~K}$ for $6 \mathrm{~h}$ in flowing air (1.67 $\mathrm{mL} \mathrm{s}^{-1}$, ultrapure air, Airgas), ion-exchanged three times using $1 \mathrm{M}$ aqueous ammonium nitrate $\left(\mathrm{NH}_{4} \mathrm{NO}_{3}\right)$ (weight ratio of zeolite to $\mathrm{NH}_{4} \mathrm{NO}_{3}$ solution $=1: 10$ ) at $353 \mathrm{~K}$ for $2 \mathrm{~h}$, and 
subsequently, collected by centrifugation, washed with DI water three times, and dried at $343 \mathrm{~K}$ overnight. All zeolite samples in their $\mathrm{NH}_{4}{ }^{+}$form were treated in air $\left(1.67 \mathrm{~mL} \mathrm{~s}^{-1}\right.$, ultrapure air, Airgas) by increasing the temperature from ambient to $823 \mathrm{~K}$ at $0.167 \mathrm{~K} \mathrm{~s}^{-1}$ rate and holding them at this temperature for $4 \mathrm{~h}$. All catalytic reactions were carried out after converting zeolites into the proton-form. The composition of $100 \mathrm{SiO}_{2} / x \mathrm{C}_{22-6-6} / 36 \mathrm{TPAOH} / 0.5 \mathrm{Na}_{2} \mathrm{O} / \mathrm{yAl}_{2} \mathrm{O}_{3} / 4000 \mathrm{H}_{2} \mathrm{O}$, where the template molar ratios $\left(\mathrm{C}_{22-6-6} / \mathrm{TPAOH}, x / 36\right)$ were $0 / 36,1 / 36,3 / 36$, and 5/36, respectively, and $y$ varied from 0.1 to 0.7 for HLBM-3/36 zeolite sample, was used for HLBM zeolite synthesis. The as-obtained zeolite samples were named after the $\mathrm{C}_{22-6-6} / \mathrm{TPAOH}$ ratio, which are HLBM-0/36, HLBM-1/36, HLBM-3/36, and HLBM-5/36, respectively. HLBM-0/36 is simply a conventional microporous MFI zeolite. For consistency in nomenclature, HLBM0/36 is designated for conventional MFI zeolite in this paper.

2.2. Characterization of HLBM zeolite catalysts. SEM images of the HLBM samples were recorded on a Hitachi SU-70 electron microscope. TEM images were collected by a JEM 2100 LaB6 electron microscope. Powder XRD patterns were recorded using a Bruker D8 Advance Lynx Powder Diffractometer (LynxEye PSD detector, sealed tube, $\mathrm{Cu} \mathrm{K}_{\alpha}$ radiation with $N i \beta$-filter) in the $2 \theta$ range from 1.25 to 40 degree. The Ar sorption isotherms were measured using an Autosorb-iQ analyzer (Quantachrome Instruments) at $87 \mathrm{~K}$. Si and $\mathrm{Al}$ contents of each sample were determined by inductively coupled plasma optical emission spectroscopy (ICP-OES, Optima 4300 DV instuments, Perkin-Elmer). The magic angle spinning NMR (MAS NMR) spectra were recorded at a field of 9.4 T (Bruker DSX 300) and $11.7 \mathrm{~T}$ (Bruker Avance 500) for ${ }^{29} \mathrm{Si}$ and ${ }^{27} \mathrm{Al}$, respectively. ${ }^{29} \mathrm{Si}$ MAS NMR spectra were recorded at $59.63 \mathrm{MHz}$ using $4 \mathrm{~mm}$ rotors at a spinning speed of $10 \mathrm{kHz}$, a dwell time of $19.95 \mu \mathrm{s}$, a $\pi / 2$ pulse of $5.0 \mu \mathrm{s}$ and a recycle delay of $60 \mathrm{~s}$. The spectra were referenced with respect to 3- 
(trimethylsilyl)-1-propanesulfonic acid salt (0 ppm). ${ }^{27} \mathrm{Al}$ MAS NMR spectra were recorded at 130.34 MHz using $4 \mathrm{~mm}$ rotors at $14 \mathrm{kHz}$ spinning speed, a dwell time of $0.5 \mu$ s, a selective $\pi / 18$ pulse of $0.3 \mu$ s and a recycle delay of $0.1 \mathrm{~s}$. An aqueous solution of aluminum sulfate $(0.1 \mathrm{M})$ was used as the reference (0 ppm). The type and concentration of Brønsted and Lewis acid sites in the HLBM catalysts were determined by FTIR spectra of adsorbed pyridine using Nicolet 6700 spectrophotometer (Thermo-Scientific) equipped with high temperature reaction chamber in conjucation with Praying Mantis ${ }^{\mathrm{TM}}$ diffuse reflection accessory (Harrick). Prior to the measurement, the zeolite sample was pretreated at $823 \mathrm{~K}$ (rate of $4.55 \mathrm{~K} \mathrm{~s}^{-1}$ ) under Ar flow of $0.83 \mathrm{~mL} \mathrm{~s}^{-1}$ for $1 \mathrm{~h}$. The catalyst sample was then cooled down to $393 \mathrm{~K}$ and exposed to pyridine by flowing Ar through pyridine saturator for $0.25 \mathrm{~h}$. After adsorption of pyridine, the sample was heated to $523 \mathrm{~K}$ (rate of $4.55 \mathrm{~K} \mathrm{~s}^{-1}$ ) under Ar flow for $1 \mathrm{~h}$ in order to desorb the physisorbed pyridine, cooled to the temperature of $393 \mathrm{~K}$, and then FTIR spectra were collected with 32 scans and at an effective resolution of $2 \mathrm{~cm}^{-1}$.

2.3. Determination of external surface, pore mouth and internal Brønsted acid sites. The $\mathrm{Si} / \mathrm{Al}$ ratio in each HLBM zeolite sample was analyzed by elemental analysis (inductively coupled plasma optical emission spectroscopy, ICP-OES). The total number of Brønsted acid sites, defined as total acid sites, was determined via dimethyl ether (DME) titration experiment given that the Brønsted acid sites participate in reactions of DME with $\mathrm{H}^{+}$to form surface methyl groups, $\mathrm{CH}_{3} \mathrm{OCH}_{3}+2[\mathrm{SiO}(\mathrm{H}) \mathrm{Al}]=2\left[\mathrm{SiO}\left(\mathrm{CH}_{3}\right) \mathrm{Al}\right]+\mathrm{H}_{2} \mathrm{O} \cdot[20,21]$ The number of active Brønsted acid sites on the external surface and in micropore mouth of zeolites, defined as external acid sites, was measured by 2,6-di-tertbutyl-pyridine (DTBP) titration during methanol dehydration reactions since the bulky DTBP molecule has limited accessibility to acid sites located in micropores of the medium-pore zeolites[22, 23]. The number of external surface acid 
sites was quantified by triphenyl phosphine (TPP) titration during methanol reaction. The sterically bulky molecular structure and moderate base strength of TPP suggest that it preferentially accesses to acid sites on the external surface of the HLBM zeolite catalysts [24-26]. The experimental setup and reaction conditions for chemical titration of acid sites were same as those described in our previous publication[21, 27, 28]. The Brønsted acid sites only accessible to DME titrant were defined as the internal acid sites. The fraction of external Brønsted acid sites was calculated by the ratio of the number of Brønsted acid sites accessible to DTBP titrant to the number of Brønsted acid sites accessible to DME titrant. Similarly, the fraction of external surface acid sites was calculated by the ratio of the number of Brønsted acid sites accessible to TPP to the number of Brønsted acid sites accessible to DME titrant. The fraction of pore mouth acid sites was calculated by the deduction of fraction of external surface acid sites from that of the external acid sites of HLBM zeolite samples.

2.4. Catalytic conversion of benzyl alcohol in mesitylene. The liquid phase catalytic conversion of benzyl alcohol (Sigma-Aldrich, 99.95\% purity) in mesitylene (Sigma-Aldrich, $99 \%$ purity) was carried out in a three-necked round bottom flask (100 mL) equipped with a reflux condenser and heated in a temperature controlled oil bath under atmospheric pressure and magnetic stirring (1" stirring bar, $500 \mathrm{rpm}$ stirring speed) conditions, similar to what reported in our previous work $[29,30]$. In a typical experiment, $15 \mathrm{ml}$ of mesitylene was added to the desired amount of zeolite catalyst, which had been activated at $823 \mathrm{~K}$ in flowing air $\left(1.67 \mathrm{~mL} \mathrm{~s}^{-1}\right)$ for $4 \mathrm{~h}$. The reaction mixture was maintained for $0.5 \mathrm{~h}$ at the required reaction temperature and stirring conditions and then $0.25 \mathrm{ml}$ of benzyl alcohol was added. This moment of benzyl alcohol addition was taken as the initial reaction time. Liquid samples were withdrawn at regular intervals and analyzed by a gas chromatograph (Agilent 7890A) equipped with a methylsiloxane 
capillary column (HP-1, $50.0 \mathrm{~m}$ x $320 \mu \mathrm{m}$ x $0.52 \mu \mathrm{m}$ ) connected to a flame ionization detector (FID) to calibrate and separate the reactants and products.

The DTBP poisoning experiment was operated under the same reaction conditions mentioned above except adding DTBP into the reactant suspension. Typically, zeolite catalyst, DTBP, and mesitylene were added to the reactor in sequence, and then the reactant mixture was held at the reaction conditions for $2.5 \mathrm{~h}$ before benzyl alcohol was added. Excess mixing time prior to benzyl alcohol addition ensured base molecules completely poisoned active sites on the geometric and mesoporous surfaces (external surfaces) of zeolite catalysts. The influence of external mass transfer limitations on the reaction rates was ruled out by running the reactions at a high enough stirring speed $(500 \mathrm{rpm})$, showing a further increase in the stirring speed did not enhance the reaction rate anymore. The reactant mixture without addition of zeolite catalysts showed no conversion of benzyl alcohol at the investigated reaction conditions.

\section{Results and discussion}

3.1. Structural properties of HLBM zeolite catalysts. The scheme for formation of HLBM silicalite zeolite has been studied in our previous publication. ${ }^{17}$ It is shown that a cooperative function between $\mathrm{TPAOH}$ and $\mathrm{C}_{22-6-6}$ templates led to formation of microporous core and lamellar shell materials, respectively, in the HLBM zeolite. The modulation of dual template ratios in the synthesis tailors the relative quantity of microporous MFI core and lamellar MFI shell, respectively, and thus the structure and textural properties oF HLBM zeolites. The synthesis of HLBM zeolite catalysts is expected to follow the same scheme since the synthesis recipe is same as that of HLBM silicalite except the addition of aluminum source. Figure 1 shows XRD patterns of the synthesized HLBM zeolite catalysts. The diffraction peaks in each 
sample resembles the characteristic of a crystalline MFI[19, 31], confirming the successful crystallization of HLBM zeolite. The diffraction peaks in the low-angle range $\left(2 \theta<7^{\circ}\right)$ of the XRD patterns indicate the evolution of the multilamellar feature of the HLBM zeolite with increasing $\mathrm{C}_{22-6-6}$ content in the dual template assisted zeolite synthesis. The morphologies of HLBM zeolite catalysts are shown by the SEM and TEM images in Figure 2. The HLBM-0/36 zeolite (Figure 2(A)-(B)) consists of short cylindrical particles with an average width of $\sim 200$ $\mathrm{nm}$ and thickness of $\sim 150 \mathrm{~nm}$ and well crystallized smooth surfaces. The HLBM-1/36 particles in Figure 2(C) have similar morphologies to those of HLBM-0/36, but they have relatively rougher crystal surfaces and larger particle sizes. A close examination of the HLBM-1/36 particles in Figure 2(D) indicates that the rough surface is composed of a lamellar structure. The SEM images in Figure 2(E) and 2(G) and TEM images in Figure 2(F) and 2(H), respectively, which correspond to HLBM-3/36 and HLBM-5/36 samples, show clearly visible lamellar shell structures. The thickness of lamellar shell in HLBM-3/36 increased compared to HLBM-1/36, and these lamellae in the shell are parallel to each other. In HLBM-5/36, however, the lamellae in the shell are incorporated into a network covered on the surface of the core zeolite. In some cases, the core seems to be dissolved, and the lamellar zeolite forms a hollow shell. The morphology transformation with increasing $\mathrm{C}_{22-6-6}$ used in the dual template synthesis suggests that relative quantity of the meso- and microporous domains can be manipulated in the HLBM zeolite samples. Based upon the respective dimensions of lamellar and bulk MFI structures in the TEM images, the mass fractions of lamellar shell in HLBM zeolite particles can be estimated if the densities of both structures are known. The density of as-made lamellar MFI is $1.54 \mathrm{~g} \mathrm{~mL}^{-1}$, while the density of calcined layered MFI is not known. From the densities of the bulk calcined MFI and as-made bulk MFI, which are $1.73 \mathrm{~g} \mathrm{~mL}^{-1}$ and $2.05 \mathrm{~g} \mathrm{~mL}^{-1}[19,32]$, respectively, the 
density of calcined lamellar MFI is estimated to be $1.54 \mathrm{x}(1.73 / 2.05)=1.30 \mathrm{~g} \mathrm{~mL}^{-1}$. The mass fractions of HLBM-1/36, HLBM-3/36 and HLBM-5/36 zeolite samples are then calculated to be $25 \%, 50 \%$ and $65 \%$, respectively. It should be noted that this is quite rough estimation since arcuate density and packing structure of lamellar shell in each HLBM zeolite is not known.

Figure 3 shows the high resolution TEM images of HLBM-1/36, HLBM-3/36, and HLBM-5/36 zeolite samples. The morphology of HLBM-1/36 (Figure 3(A)-(C)), HLBM-3/36 (Figure 3(D)-(F)) and HLBM-5/36 (Figure $(\mathrm{G})$ and $(\mathrm{H})$ ) is similar to porcupine sensory message ball with length of little nerve-stimulating bumps increased from HLBM-1/36 to HLBM-5/36. The lattice fringes of the mesoporous lamellar shell are clearly visible in these TEM images which indicate the mesoporous shell of the HLBM zeolite is crystallized. Figure 3 also suggests that microporous and mesoporous structures in HLBM zeolite are organized and interconnected. This unique morphology offers a pathway for tailoring the physicochemical properties of hierarchical meso-/microporous zeolite materials in the area of catalysis.

3.2. Textural properties of HLBM zeolite catalysts. The textural properties of HLBM zeolites were analysed by the Ar adsorption-desorption isotherms, as shown in Figure 4. As expected, the external surface area of the HLBM zeolites increases with increasing $\mathrm{C}_{22-6-6}$ content in the zeolite synthesis (Figure 4(A)). Non-local density functional theory (NLDFT) pore size analysis was derived from the adsorption branch of $\mathrm{Ar}$ isotherms on the basis of spherical/cylindrical pore model. Figure 4(B) illustrates the presence of dual micropore and mesopore systems in HLBM zeolite and the mesopore sizes increase from HLBM-0/36 to HLBM-5/36 samples. Table 1 summarizes the surface areas and pore volumes of the HLBM zeolite. The external surface area, mesopore volume, and total pore volume increase by increasing the $\mathrm{C}_{22-6-6}$ content in the zeolite synthesis. This is caused by the production and 
growth of the mesoporous lamellar shell in HLBM zeolite structures. The micropore volume and micropore surface area, however, show opposite trend with $\mathrm{C}_{22-6-6}$ content in the synthesis. The decrease in micropore volume and micropore surface area upon mesostructuring a zeolite material in the present study is consistent with those reported for mesoporous zeolite prepared by demetalization [33, 34] or pillaring of layered zeolite nanosheets[35]. The generation of mesoporosity in the zeolite samples seems to sacrifice slightly the microporosity of zeolite materials.

It should be noted that the $\mathrm{Si} / \mathrm{Al}$ ratios in the dual template assisted synthesis influenced the morphology of the resultant HLBM zeolite. By lowering the Si/Al ratio, the long-range ordering of the lamellae shell in HLBM zeolite is slowly diminished, as shown by the SEM images in Figure S1 of the Supplementary Information. The textural properties of HLBM zeolite samples, however, are not influenced obviously by changing $\mathrm{Si} / \mathrm{Al}$ ratios in the synthesis, as indicated by similar surface areas and pore volumes of the HLBM-3/36 samples with different $\mathrm{Si} / \mathrm{Al}$ ratios in Table S1 of the Supplementary Information.

3.3. Acidity properties of HLBM zeolite catalysts. Table 2 lists the $\mathrm{Si} / \mathrm{Al}$ ratios and the number of Brønsted acid sites of HLBM zeolite samples determined by ICP-OES measurement and DME titration, respectively. The number of external active sites of each catalyst was determined from DTBP titrations, and is also shown in Table 2. The fractions of external acid sites $\left(f_{\mathrm{B}, \mathrm{ext}}\right)$ were calculated by the ratio of the number of Brønsted acid sites determined from DTBP titration to the number of Brønsted acid sites determined from DME titration. It is known that the bulky DTBP molecules have limited accessibility to Brønsted acid sites located in micropores in MFI-type zeolites[22, 23]. The increase in fractions of external acid sites from HLBM-0/36 to HLBM-5/36 reflects that the acid sites associated with framework $\mathrm{Al}$ is spatially 
modulated between the mesoporous lamellar shell and microporous bulk core domains in HLBM zeolites.

The external acidity commonly results from acid sites located on the external surface and at the pore mouth region of the hierarchical zeolite catalysts[36-38]. The creation of mesoporosity or delamination in HLBM zeolites increases the number of acid sites both in the pore mouth region and on the external surface compared to the microporous MFI zeolite. To identify the locations (on the external surface or in the pore mouth region) of external acid sites in HLBM zeolites, the organic base titration with TPP molecule was conducted. TPP titrant has moderate base strength and bulky molecular structure, and thus it is expected to anchor on the external surface acid sites [24-26]. The difference between the number of acid sites determined by DTBP and TPP titrations indicates the number of acid sites located in the pore mouth region of the catalysts. Additionally, silynation of external acid sites of HLBM zeolites by ALD of TDMAS precursor followed by DTBP titration was also conducted to analyze the external surface and pore mouth acid sites in HLBM zeolites. The bulky TDMAS cannot enter the micropores of MFI zeolite, but preferentially stays at the pore mouth region, similar to that of silynation of zeolite with tetraethyl orthosilicate precursor [39-41]. The DTBP titration of HLBM zeolites after $\mathrm{ALD}-\mathrm{SiO}_{2}$ modifications determines the number of external surface acid sites, while the difference from that determined from DTBP titration in the absence of $\mathrm{ALD}-\mathrm{SiO}_{2}$ modification suggests the number of pore mouth acid sites. Table 3 shows the fractions of external surface and pore mouth acid sites determined by both methods. Clearly, the number of acid sites in the pore mouth region is lower than that on the external surface of HLBM zeolites. Both types of external acid sites increase with varying the MFI zeolite structure from HLBM- 
0/36 to HLBM-5/36, similar to those obtained from DTBP titration of HLBM zeolites discussed above.

Solid state NMR was employed to investigate the local bonding environment in the synthesized HLBM zeolite catalysts. Figure $5(\mathrm{~A})$ shows the ${ }^{29} \mathrm{Si}$ single pulse (SP) NMR spectra of HLBM-1/36, HLBM-3/36, and HLBM-5/36 zeolite samples. Two resolved peaks (at -113 and $-103 \mathrm{ppm}$ ) can be observed in the ${ }^{29} \mathrm{Si}$ SP NMR spectrum of the MFI zeolite, which correspond to crystallographically nonequivalent $\mathrm{Q}^{4}$ tetrahedral sites $\left(\mathrm{Q}^{\mathrm{n}}\right.$ stands for $\left.\mathrm{X}_{4-\mathrm{n}} \mathrm{Si}[\mathrm{OSi}]_{\mathrm{n}}\right)[40,42-44]$ and $\mathrm{Q}^{3}$ sites arising from the silanol groups on the zeolite surface[45]. The similar $\mathrm{Q}^{4}$ and $\mathrm{Q}^{3}$ peaks in these catalysts to those reported for pillared MFI zeolite[21] suggest that there is a similar local bonding environment in these meso-/microporous HLBM and pillared MFI zeolites. Figure 5(B) shows the ${ }^{27} \mathrm{Al}$ MAS NMR spectra of the HLBM zeolite samples. The peak at 55 ppm is due to the tetrahedrally coordinated framework aluminum $\left(\mathrm{Al}_{\mathrm{F}}\right)[21,43]$, while the peak around $0 \mathrm{ppm}$ is due to an octahedral coordination typical of extra-framework $\mathrm{Al}\left(\mathrm{Al}_{\mathrm{EF}}\right)$. The fractions of the extra-framework $\mathrm{Al}$ in these HLBM zeolite samples should be insignificant quantity since the peak around $0 \mathrm{ppm}$ is very small compared to that around $55 \mathrm{ppm}$ in the spectra.

The type and concentration of acid sites were evaluated by FTIR spectra of the adsorbed pyridine on the HLBM zeolite catalysts, and the results are shown in Figure 6. The absorption band at $1545 \mathrm{~cm}^{-1}$ is typical of pyridine interacting with Brønsted acid sites, while the absorption band around $1455 \mathrm{~cm}^{-1}$ is assigned to the interaction of pyridine with Lewis acid sites in the zeolite catalysts.[46, 47] Figure 6(A) shows that $1545 \mathrm{~cm}^{-1}$ and $1455 \mathrm{~cm}^{-1}$ bands exist in the spectra of all the HLBM zeolite samples, indicating the co-existence of Brønsted and Lewis acid sites in these catalysts. The concentrations of the individual Brønsted and Lewis acid sites were 


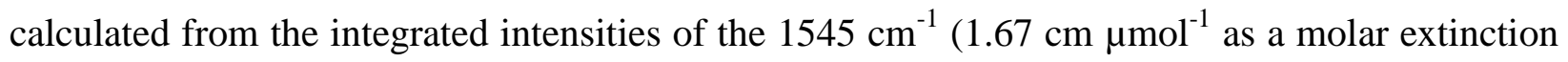

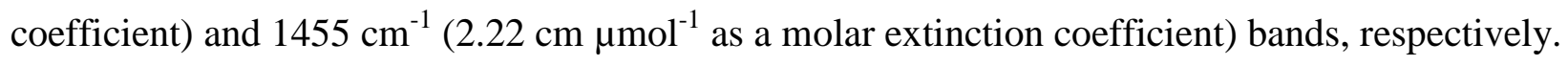
Table 2 shows the molar ratios of Brønsted-to-Lewis (B/L) acid site for these four HLBM zeolite catalysts. The B/L acid site ratio decreases from HLBM-0/36 to HLBM-5/36, which reveals that modulation in morphological and textural properties of the HLBM catalysts intensely influence their acidity properties. A correlation between the $\mathrm{B} / \mathrm{L}$ acid site ratio and the relative external surface area $\left(\mathrm{S}_{\mathrm{ext}} / \mathrm{S}_{\mathrm{BET}}\right)$ of HLBM zeolite samples is shown in Figure 6(B). The B/L acid site ratio increases with decreasing the relative external surface area of the HLBM catalysts, clearly illustrating the influence of spatially controlled morphology on the acidity of HLBM zeolite. The increase in Levis acidity from HLBM-0/36 to HLBM-5/36 is resulted from the increasing quantity of lamellar shell structures. It has been reported that the two-dimensional zeolite structures possess increased amount of Lewis sites due to the missing connectivities in the third dimension of the zeolite structure.[34, 48, 49]

3.4. Catalytic performance of HLBM zeolite catalysts. The liquid phase catalytic conversion of benzyl alcohol in mesitylene, reaction scheme shown in section S2 of the Supplementary Information, was employed to examine the catalytic consequences for the selectivity of external acidity in meso-/microporous HLBM zeolites. The parallel reactions, alkylation and etherification, of benzyl alcohol produce 2,4,6-trimethylbiphenyl and dibenzyl ether products, respectively. The series reactions convert the reaction intermediate, dibenzyl ether, to the alkylation product, 2,4,6-trimethylbiphenyl.[13] The kinetic diameter of mesitylene $(0.87 \mathrm{~nm})$ is apparently larger than micropore sizes of MFI zeolites [50]. It is expected that the alkylation reaction exclusively occurs on the external surface acid sites of the HLBM zeolites. The apparent rate constants of the parallel reactions over the meso-/microporous HLBM catalysts 
were evaluated at $348 \mathrm{~K}$, excess amount of mesitylene (molar ratio of mesitylene to benzyl alcohol $=45: 1)$, rigorous agitation, and less than $10 \%$ conversion of benzyl alcohol to eliminate the influences of catalyst deactivation, external mass transfer limitation, or deficiency of dibenzyl ether product due to series reactions.

Figure 7 (A) and (B) show the measured rate constants for conversion of benzyl alcohol in the parallel alkylation and etherification reactions over meso-/microporous HLBM zeolite catalysts under conditions where all active sites of the zeolite catalysts were unperturbed (Figure 7(A)) and perturbed (Fiure 7(B), by DTBP titrant), respectively. The measured rate constants were obtained by simply fitting the data using a pseudo-first order rate law. In the absence of the 2,6-di-tert-butylpyridine (DTBP) titrant, the HLBM zeolites had an increasing alkylation rate while decreasing etherification rate with increasing $\mathrm{C}_{22-6-6} / \mathrm{TPAOH}$ ratios employed in the catalyst synthesis. The selectivity $\left(\alpha=2 \mathrm{C}_{\mathrm{E}} / \mathrm{C}_{\mathrm{C}}, \mathrm{C}_{\mathrm{E}}\right.$ and $\mathrm{C}_{\mathrm{C}}$ are the concentrations of dibenzyl ether and 1,3,5-trimethyl-2-benzylbenzene in solution $\left(\mathrm{mol} \mathrm{L}^{-1}\right)$, respectively) of the parallel reactions is listed in Table S2 of the Supplementary Information, indicating alkylation reaction was favored with increasing mesoporous domain in the HLBM zeolites. In addition, the selectivity differs by $\sim 2$ order of magnitude across these four HLBM zeolite catalysts. The consequences for the remarkably difference in selectivity of the parallel reactions were explored using DTBP titration to selectively poison the acid sites on the external environment (external surface acid sites and acid sites in pore mouth region) of catalysts since the steric resistance hinders the access of bulky DTBP molecules to acid sites located in micropores of HLBM zeolites. In the presence of DTBP titrant, HLBM zeolite samples maintained the residual activity for etherification reaction as shown in Figure 7(B), suggesting that the etherification reaction occurs on both internal and external environments of HLBM catalysts although the alkylation 
reaction exclusively happens on the external surface environment of HLBM catalysts. The parallel reaction was also carried out over HLBM zeolites after ALD-SiO $\mathrm{S}_{2}$ modifications. Similar alkylation rate and slightly lower etherification reaction rate were observed compared to results present in Figure 7(A), suggesting that acid sites in pore mouth region were active for etherification reaction but not involved in alkylation reaction. The selective poisoning of external surface acid sites of HLBM zeolites was conducted by addition of TPP in the parallel reactions. The alkylation reaction disappeared and similar etherification rates to those in Figure 7(B) were observed from this test. These results also indicate that alkylation takes place on the external surface acid sites and etherification reaction occurs over external surface acid sites, acid sites in pore mouth and acid sites in micropore of HLBM zeolites. The explicit elucidation of catalytic performance of these three types of acid sites in etherification reaction requires further analysis of effects of internal diffusion in the HLBM zeolites, in which complexity of the reactiondiffusion data analysis is beyond the scope of the present study. Therefore, we simply limit our analysis to external surface acid sites in alkylation reaction, external acid sites (on external surface and in pore mouth region) in etherification reaction, and internal acid sites in etherification reaction, respectively, as discussed below.

The involvement of acid sites on external and internal environments of HLBM zeolites in catalyzing parallel alkylation and etherification reactions of benzyl alcohol in mesitylene is therefore systematically evaluated by correlating the reactions occurring at each location to their corresponding rate constants evaluated from catalytic results in the absence and presence of DTBP titrant, respectively. The rate constants can be separated into $\mathrm{k}_{\mathrm{alk}, \mathrm{ext}}$ surf (per external surface Brønsted acid site, $[\mathrm{mol} \mathrm{H}]^{+} \mathrm{s}^{-1}$ ), $\mathrm{k}_{\text {eth,ext }}$ (per external Brønsted acid site, $\left[\mathrm{mol} \mathrm{H}^{+}\right]^{-1} \mathrm{~s}^{-1}$ ), and $\mathrm{k}_{\text {eth,int }}$ (per internal Brønsted acid site, $\left[\mathrm{mol} \mathrm{H}^{+}\right]^{-1} \mathrm{~s}^{-1}$ ), corresponding to the alkylation reaction 
on the external surface environment, etherification on the external environment (external surface acid site and acid site in pore mouth region), and etherification in the internal microporous environment, respectively. The alkylation reaction data were fitted well to a pseudo-first order rate law: $\ln \frac{C_{\mathrm{A}}^{0}}{C_{\mathrm{A}}}=k_{\text {alk,ext surf }} M_{\mathrm{B}} f_{\mathrm{B}, \text { ext surf }}(1+\alpha) t=k_{\text {alk,app }} t$, where $C_{A}^{0}$ is the initial benzyl alcohol concentration in solution $\left(\mathrm{mol} \mathrm{L}^{-1}\right), C_{A}$ is benzyl alcohol concentration in solution $\left(\mathrm{mol} \mathrm{L}^{-1}\right), M_{\mathrm{B}}$ $\left(\mathrm{mol} \mathrm{H}^{+}\right)$is the total moles of Brønsted acid sites present in the batch reactor, $f_{\mathrm{B}, \mathrm{ext} \text { surf }}$ is the external surface Brønsted acid site fraction (Table 3), and $t$ is the reaction time (s), respectively, to solve the apparent rate constants. Figure 7(C) shows that $k_{\text {alk,ext surf }}$ increases by increasing the relative external surface area of the HLBM zeolite catalysts. The internal reaction rate ( $\left.\mathrm{k}_{\text {eth,int }}\right)$ coupled with internal mass transport limitations on HLBM zeolites can be rigorously described by a Thiele Modulus treatment, as our previous work[29, 30] on treatment of zeolites with different crystallite sizes. The catalytic activity of unit-cell thick pillared MFI and self-pillared MFI is presumed as the intrinsic kinetic behavior. By employing $\mathrm{D}_{\mathrm{A}}$ (diffusion coefficient of benzyl alcohol in the catalysts $\left(\mathrm{m}^{2} \mathrm{~s}^{-1}\right)$, evaluated from $17 \mu \mathrm{m}$ MFI in our previous work[29]) and the intrinsic catalytic rate behavior, the effectiveness factor ( $\eta$ ) of each HLBM catalysts can be evaluated. The difference in effectiveness factor (Table S3) across the HLBM zeolite catalysts indicates they have different internal etherification catalytic behaviors.

We aim to compare the etherification and alkylation rate constants on the external environment of HLBM zeolite catalysts. Although alkylation rates correspond to external surface acid sites, etherification rates contain two contributions, one from external sites (external surface and pore mouth) and one from reaction inside the micropores (internal) as discussed above. In order to obtain external etherification rates for HLBM catalysts, the contribution of internal 
etherification rates should be subtracted. The later can be measured independently by deactivating the external active sites with DTBP titration and therefore, deconvolution of the two contributions to the overall rate can be accomplished. The analysis presented in detail in S3.2 of the Supplementary Information provides $k_{\text {eth,ext }}$ for each HLBM zeolite catalyst (Table S4). Figure 7(D) shows the rate constants of external etherification reaction decreases with $\mathrm{S}_{\text {ext }} / \mathrm{S}_{\mathrm{BET}}$

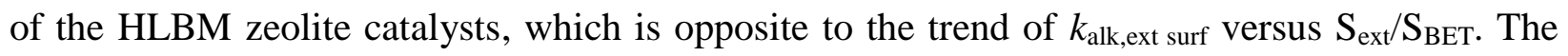
selectivity to etherification versus alkylation on external acid sites, defined as $\alpha^{\prime}=\frac{k_{\text {eth,ext }}}{k_{\text {alk,extsuf }}}$ (Figure 7(E) and Table S4), consistently decreases with increasing relative external surface area of the HLBM catalysts. The results and analysis presented here demonstrate substantial differences of external surface catalytic behavior of the HLBM zeolite catalysts.

In order to understand further on the catalytic behavior of the external acid sites in HLBM zeolites, we conducted the experiments to accumulatively poison the external acid sites of HLBM-3/36 and HLBM-5/36 in the benzyl alcohol reactions by controlled addition of DTBP during the reaction. Figure $8(\mathrm{~A})$ shows that $\mathrm{k}_{\text {eth,ext }}$ (per remaining active external acid sites) is close to a constant with the increasing number of external active sites poisoned in both HLBM3/36 and HLBM-5/36 catalysts, suggesting that etherification exclusively occurred on external environment of catalysts is not influenced significantly by the type or strength of the external active sites. Figure $8(\mathrm{~B})$ demonstrates that $\mathrm{k}_{\mathrm{alk}}$,ext surf (per remaining external surface active acid sites) decreases with continuous addition of DTBP to poison the external surface acid sites, indicating that external surface acid sites responded differently for the external surface alkylation reaction from external etherification reaction. The prompt drop in alkylation activity (> 50\%) with a small fraction of external surface acid site $(\sim 10 \%)$ poisoned and the slow drop in activity with a large fraction of external poisoned acid sites imply that some of the individual external 
surface acid sites are very active while others are insignificant in enabling the alkylation reaction, which might result from the different acid strengths of the external surface acid sites. Additionally, the steric resistance from adsorbed DTBP on external surface acid sites might contribute to the non-linear activity loss behavior in Figure 8(B). The cumulative poisoning experiment suggests that individual external surface acid sites in HLBM zeolites contribute differently to external surface alkylation reaction.

\section{Conclusions}

A scenario of catalytic reactions of benzyl alcohol in mesitylene over the meso/microporous HLBM zeolite catalysts has been elucidated based on the catalytic results in the absence and presence of DTBP titrant together with the tunable dual meso-/microporosity of the zeolites. The distinct selectivity differences in parallel etherification and alkylation of benzyl alcohol in mesitylene across the HLBM zeolites are attributed to the different external acid site environments that catalyzed reactions with variant rates in HLBM zeolites. The measured rate behavior of etherification and alkylation reactions on external environment of HLBM zeolites scales with the relative external surface area and B/L acidity of the catalysts. A systematic study of HLBM zeolites with cumulative poisoning external acid sites revealed that alkylation reaction rate (per remaining external surface active acid site) on external environment monotonically decreased with increasing number of acid sites poisoned on the external surface areas of HLBM zeolites. The external etherification reaction rate (per remaining external active acid site) was not influenced significantly by the cumulative poisoning of the catalysts. The present study exemplified for the first time that the external acidity in meso-/microporous HLBM zeolites is vital in impacting the selectivity of the parallel catalytic reactions. 


\section{Acknowledgements}

The authors gratefully acknowledge financial support from the ACS-Petroleum Research Fund (ACS-PRF) and National Science Foundation (NSF-CBET 1264599). We acknowledge the support of Maryland NanoCenter and its NispLab. The NispLab is supported in part by the NSF as a MRSEC Shared Experimental Facility.

Supplementary information available: The morphology and textural properties of HLBM-3/36 zeolite samples with different $\mathrm{Si} / \mathrm{Al}$ ratios; the derivation of apparent rate constants from the benzyl alcohol conversion data using a pseudo-first order rate law; and analysis for catalytic etherification under internal mass transfer limitations are presented.

\section{References}

[1] J. Čejka, S. Mintova, Cat. Rev. - Sci. Eng., 49 (2007) 457.

[2] J. Perez-Ramirez, C.H. Christensen, K. Egeblad, C.H. Christensen, J.C. Groen, Chem. Soc. Rev., 37 (2008) 2530.

[3] J. Zhu, X. Meng, F. Xiao, Front. Chem. Sci. Eng., 7 (2013) 233.

[4] W.J. Roth, P. Nachtigall, R.E. Morris, J. Cejka, Chem. Rev., 114 (2014) 4807.

[5] J. C. Kim, R. Ryoo, M.V. Opanasenko, M.V. Shamzhy, J. Cejka, ACS Catal., 5 (2015) 2596.

[6] K. Na, G.A. Somorjai, Catal. Lett., 145 (2015) 193.

[7] C.S. Cundy, P.A. Cox, Chem. Rev., 103 (2003) 663.

[8] K. Egeblad, C.H. Christensen, M. Kustova, C.H. Christensen, Chem. Mater., 20 (2008) 946.

[9] W. Fan, M.A. Snyder, S. Kumar, P. S. Lee, W.C. Yoo, A.V. McCormick, R. L. Penn, A. Stein, M. Tsapatsis, Nat. Mater., 7 (2008) 984.

[10] Guest editors: B. Lebeau, A. Galarneau, M. Linden, Themed collection: Mesoporous Materials., Chem. Soc. Rev., 42 (2013) 3649.

[11] Guest editors: B. Weckhuysen, Ji. Yu, Themed collection: Recent Advances in Zeolite Chemistry and Catalysis, Chem. Soc. Rev., (2015) (10 advanced articles).

[12] Y. Li, H.M. Sun, Y.H. Wang, B.J. Xu, Z.F. Yan, Prog. Chem., 27 (2015) 503.

[13] N. Narender, K.V.V. Krishna Mohan, S.J. Kulkarni, I. Ajit Kumar Reddy, Catal. Comm., 7 (2006) 583.

[14] Y. Sun, R. Prins, Appl. Catal. A., 336 (2008) 11.

[15] F. Thibault-Starzyk, I. Stan, S. Abelló, A. Bonilla, K. Thomas, C. Fernandez, J.-P. Gilson, J. Pérez-Ramírez, J. Catal., 264 (2009) 11.

[16] X. Li, R. Prins, J.A.v. Bokhoven, J. Catal., 262 (2009) 257.

[17] L. Emdadi, D.X. Liu, J. Mater. Chem. A, 2 (2014) 13388.

[18] L. Emdadi, Y. Q. Wu, G. Zhu, C. C. Chang, W. Fan, T. Pham, R.F. Lobo, D. X. Liu, Chem. Mater., 26 (2014) 1345.

[19] W.G. Kim, X. Y. Zhang, J. S. Lee, M. Tsapatsis, S. Nair, ACS Nano, 6 (2012) 9978. 
[20] P. Cheung, A. Bhan, G. J. Sunley, D. J. Law, E. Iglesia, J. Catal., 245 (2007) 110.

[21] D.X. Liu, A. Bhan, M. Tsapatsis, S. Al Hashimi, ACS Catal., 1 (2011) 7.

[22] D. Fărcaşiu, R. Leu, A. Corma, J. Phys. Chem. B, 106 (2002) 928.

[23] J. Macht, C.D. Baertsch, M. May-Lozano, S.L. Soled, Y. Wang, E. Iglesia, J. Catal., 227 (2004) 479.

[24] H.L. Ngo, E. Hoh, T.A. Foglia, Eur. J. Lipid Sci. Technol., 114 (2012) 213.

[25] B. Hu, I.D. Gay, Langmuir, 15 (1999) 477.

[26] K. Na, C. Jo, J. Kim, K. Cho, J. Jung, Y. Seo, R.J. Messinger, B.F. Chmelka, R. Ryoo, Science, 333 (2011) 328.

[27] Y.Q. Wu, L. Emdadi, Z. P. Wang, W. Fan, D. X. Liu, Appl. Catal., A, 470 (2014) 344.

[28] Y.Q.Wu, L. Emdadi, S.C. Oh, M. Sakbodin, D.X. Liu, J. Catal., 323 (2015) 100.

[29] X.Y. Zhang, D.X. Liu, D. D. Xu, S. Asahina, K.A. Cychosz, K.V. Agrawal, Y. Al Wahedi, A. Bhan, S. Al Hashimi, O. Terasaki, M. Thommes, M. Tsapatsis, Science, 336 (2012)1684.

[30] D.X. Liu, X.Y. Zhang, A. Bhan, M. Tsapatsis, Micropor. Mesopor. Mater., 200 (2014) 287.

[31] K.N. Minkee Choi, J. Kim, Y. Sakamoto, O. Terasaki, R. Ryoo, Nature, 461 (2009) 246.

[32] D.W. Breck, Zeolite Molecular Sieves: Structure, Chemistry and Use, in, John Wiley \& Sons: New York., 1974.

[33] M. Thommes, S. Mitchell, J. Pérez-Ramírez, J. Phys. Chem. C, 116 (2012) 18816.

[34] M. Milina, S. Mitchell, N.L. Michels, J. Kenvin, J. Pérez-Ramírez, J. Catal., 308 (2013)398.

[35] M.C. Kyungsu Na, W. Park, Y. Sakamoto, O. Terasaki, R. Ryoo, J. Am. Chem. Soc., 132 (2010) 4169.

[36] F.-F. Wei, Z.-M. Cui, X.-J. Meng, C.-Y. Cao, F.-S. Xiao, W.G. Song, ACS Catal., 4 (2014) 529.

[37] S.C.C. Wiedemann, A. Muñoz-Murillo, R. Oord, T. van Bergen-Brenkman, B. Wels, P.C.A. Bruijnincx, B.M. Weckhuysen, J. Catal., 329 (2015) 195.

[38] J. Martins, E. Birot, E. Guillon, F. Lemos, F.R. Ribeiro, P. Magnoux, S. Laforge, Micropor. Mesopor. Mater., 171 (2013) 238.

[39] O. Ocakoglu, C. Ustundag, N. Koyluoglu, V. Oguz, G. Kendiroglu, S. Ozkan, Curr. Eye Res., 26 (2003) 277.

[40] R. Aiello, F. Crea, F. Testa, G. Demortier, P. Lentz, M. Wiame, J.B. Nagy, Micropor. Mesopor. Mater., 35-6 (2000) 585.

[41] R.W. Weber, K.P. Möller, M. Unger, C.T. O'Connor, Micropor. Mesopor. Mater., 23 (1998) 179.

[42] S.L. Burkett, M.E. Davis, J. Phys. Chem., 98 (1994) 4647.

[43] W. Kolodziejski, C. Zicovichwilson, A. Corma, J. Phys. Chem., 99 (1995) 7002.

[44] M.A. Camblor, A. Corma, M.J. Diaz-Cabanas, C. Baerlocher, J. Phys. Chem. B, 102 (1998) 44.

[45] S. Maheshwari, C. Martínez, M.T. Portilla, F.J. Llopis, A. Corma, M. Tsapatsis, J. Catal., $272(2010) 298$.

[46] C.A. Emeis, J. Catal., 141 (1993) 347.

[47] M. Guisnet, P. Ayrault, C. Coutanceau, M. Fernanda Alvarez, J. Datka, J. Chem. Soc.Faraday Trans., 93 (1997) 1661.

[48] C. Jo, R. Ryoo, N. Žilková, D. Vitvarová, J. Čejka, Catal. Sci. Tech., 3 (2013) 2119.

[49] S. Laforge, P. Ayrault, D. Martin, M. Guisnet, Appl. Catal., A., 279 (2005) 79.

[50] V.R. Choudhary, V.S. Nayak, T.V. Choudhary, Ind. Eng. Chem. Res., 36 (1997) 1812. 


\section{List of Tables:}

Table 1. Textural properties of the HLBM zeolite catalysts determined from Ar isotherms.

\begin{tabular}{|c|c|c|c|c|c|c|}
\hline Catalyst & $\begin{array}{c}\mathrm{V}_{\text {micro }}^{a} \\
{\left[\mathrm{~cm}^{3} \mathrm{~g}^{-1}\right]}\end{array}$ & $\begin{array}{c}\mathrm{S}_{\text {micro }}{ }^{a} \\
{\left[\mathrm{~m}^{2} \mathrm{~g}^{-1}\right]}\end{array}$ & $\begin{array}{c}\mathrm{S}_{\mathrm{ext}}{ }^{a} \\
{\left[\mathrm{~m}^{2} \mathrm{~g}^{-1}\right]}\end{array}$ & $\begin{array}{c}\mathrm{V}_{\mathrm{t}}^{b} \\
{\left[\mathrm{~cm}^{3} \mathrm{~g}^{-1}\right]}\end{array}$ & $\begin{array}{c}\mathrm{V}_{\text {meso }}=\mathrm{V}_{\mathrm{t}}-\mathrm{V}_{\text {micro }} \\
{\left[\mathrm{cm}^{3} \mathrm{~g}^{-1}\right]}\end{array}$ & $\begin{array}{c}\mathrm{S}_{\mathrm{BET}}{ }^{c} \\
{\left[\mathrm{~m}^{2} \mathrm{~g}^{-1}\right]}\end{array}$ \\
\hline HLBM-0/36 & 0.127 & 310 & 130 & 0.540 & 0.413 & 441 \\
\hline HLBM-1/36 & 0.094 & 258 & 187 & 0.571 & 0.477 & 445 \\
\hline HLBM-3/36 & 0.088 & 224 & 258 & 0.690 & 0.602 & 481 \\
\hline HLBM-5/36 & 0.069 & 187 & 334 & 0.763 & 0.694 & 521 \\
\hline
\end{tabular}

${ }^{a}$ Determined from t-plot method; ${ }^{b}$ Determined by NLDFT method; ${ }^{c}$ Determined from Brunauer, Emmett, and Teller (BET) method.

Table 2. Si/Al ratio and acidity of HLBM zeolite catalysts.

\begin{tabular}{|c|c|c|c|c|c|c|}
\hline Catalyst & $\begin{array}{l}\mathrm{Si} / \mathrm{Al} \\
\text { ratio }\end{array}$ & $\begin{array}{c}\text { Total } \mathrm{Al} \\
\text { sites } \\
\left(\mathrm{x} 10^{5}, \mathrm{~mol}\right. \\
\left.\mathrm{H}^{+} \mathrm{g}^{-1}\right)\end{array}$ & $\begin{array}{c}\text { Total Brønsted } \\
\text { acid sites }{ }^{b} \\
\left(\times 10^{5}, \mathrm{~mol} \mathrm{H}^{+} \mathrm{g}^{-1}\right) \\
\end{array}$ & $\begin{array}{c}\text { External Brønsted } \\
\text { acid sites }{ }^{c} \\
\left(\mathrm{x} 10^{5}, \mathrm{~mol} \mathrm{H}^{+} \mathrm{g}^{-1}\right)\end{array}$ & $\begin{array}{c}f_{\mathrm{B}, \mathrm{ext}}{ }^{d} \\
(\%)\end{array}$ & $\begin{array}{l}\mathrm{B} / \mathrm{L} \\
\text { acid site } \\
\text { ratio }^{e}\end{array}$ \\
\hline HLBM-0/36 & 50 & 2.22 & 2.14 & 0.09 & 4 & 5.64 \\
\hline HLBM-1/36 & 55 & 2.01 & 2.30 & 0.18 & 8 & 5.22 \\
\hline HLBM-3/36 & 52 & 2.13 & 2.09 & 0.25 & 12 & 4.88 \\
\hline HLBM-5/36 & 64 & 1.74 & 1.74 & 0.28 & 16 & 3.58 \\
\hline
\end{tabular}

${ }^{a}$ Determined from elemental analysis (ICP-OES); ${ }^{b}$ Determined by DME titration; ${ }^{c}$ Determined by DTBP titration; ${ }^{d}$ The fraction of external Brønsted acid sites calculated by (number of Brønsted acid sites by DTBP titration / number of Brønsted acid sites by DME titration); ${ }^{e}$ Calculated from FTIR spectra of adsorbed pyridine.

Table 3. External acidity (external surface and in the pore mouth region) of HLBM zeolite catalysts.

\begin{tabular}{|c|c|c|c|c|c|c|}
\hline \multirow{2}{*}{ Catalyst } & \multicolumn{2}{|c|}{$\begin{array}{c}\text { External acidity determined } \\
\text { by TPP titration }{ }^{a}\end{array}$} & \multicolumn{2}{|c|}{$\begin{array}{c}\text { External acidity determined by } \\
\text { ALD-SiO }\end{array}$} & \multicolumn{2}{|c|}{$\begin{array}{c}\text { Average value of external } \\
\text { acidity }\end{array}$} \\
\cline { 2 - 7 } & $\begin{array}{c}f_{B, \text { ext surf }}{ }^{a} \\
(\%)\end{array}$ & $\begin{array}{c}f_{B, \text { pore mouth }}{ }^{6} \\
(\%)\end{array}$ & $\begin{array}{c}f_{B, \text { ext surf }}{ }^{c} \\
(\%)\end{array}$ & $\begin{array}{c}f_{B, \text { pore mouth }}{ }^{c} \\
(\%)\end{array}$ & $\begin{array}{c}f_{B, \text { ext surf }}{ }^{e} \\
(\%)\end{array}$ & $\begin{array}{c}f_{B, \text { pore mouth }}{ }^{f} \\
(\%)\end{array}$ \\
\hline HLBM-0/36 & 2.8 & 1.2 & 2.1 & 1.9 & 2.5 & 1.5 \\
\hline HLBM-1/36 & 5.2 & 2.8 & 4.8 & 3.2 & 5.0 & 3.0 \\
\hline HLBM-3/36 & 8.7 & 3.3 & 8.5 & 3.5 & 8.6 & 3.4 \\
\hline HLBM-5/36 & 11.8 & 4.2 & 10.7 & 5.3 & 11.3 & 4.8 \\
\hline
\end{tabular}

${ }^{a}$ Determined from TPP titration experiment; ${ }^{b}$ Calculated by $f_{\text {pore mouth }}{ }^{b}=f_{B \text {,ext }}-f_{B \text {,ext surf }}{ }^{a}$, where $f_{B \text {,ext }}$ was determined by DTBP titration; ${ }^{c}$ Determined by DTBP titration of HLBM zeolites after ALD-SiO ${ }_{2}$ modification; ${ }^{d}$ Calculated by $f_{B \text {, pore mouth }}{ }^{d}=f_{B, \text { ext }}-f_{B \text {,ext surf }}{ }^{c} ;{ }^{e}$ Calculated by $f_{B \text {,ext surf }}{ }^{e}=\left(f_{B \text {,ext surf }}{ }^{a}+f_{B \text {,ext surf }}{ }^{c}\right) / 2 ;{ }^{e}$ Calculated by $f_{B \text {, pore }}$ mouth $^{f}=\left(f_{B \text {, pore mouth }}{ }^{b}+f_{B \text {, pore mouth }}{ }^{d}\right) / 2$. 


\section{List of Figures:}

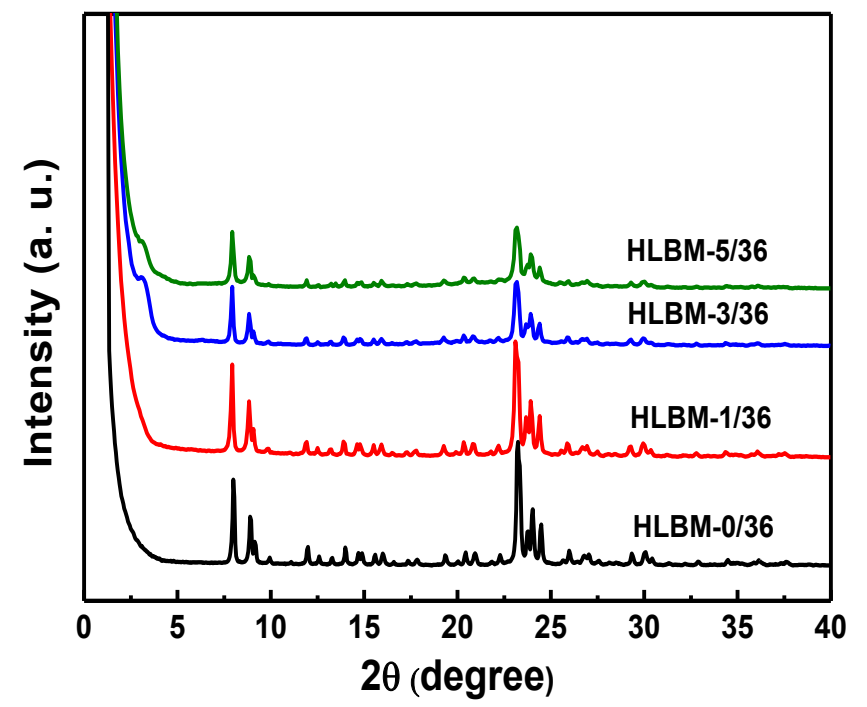

Figure 1. XRD patterns of synthesized HLBM-0/36, HLBM-1/36, HLBM-3/36, and HLBM$5 / 36$ zeolite catalysts. 

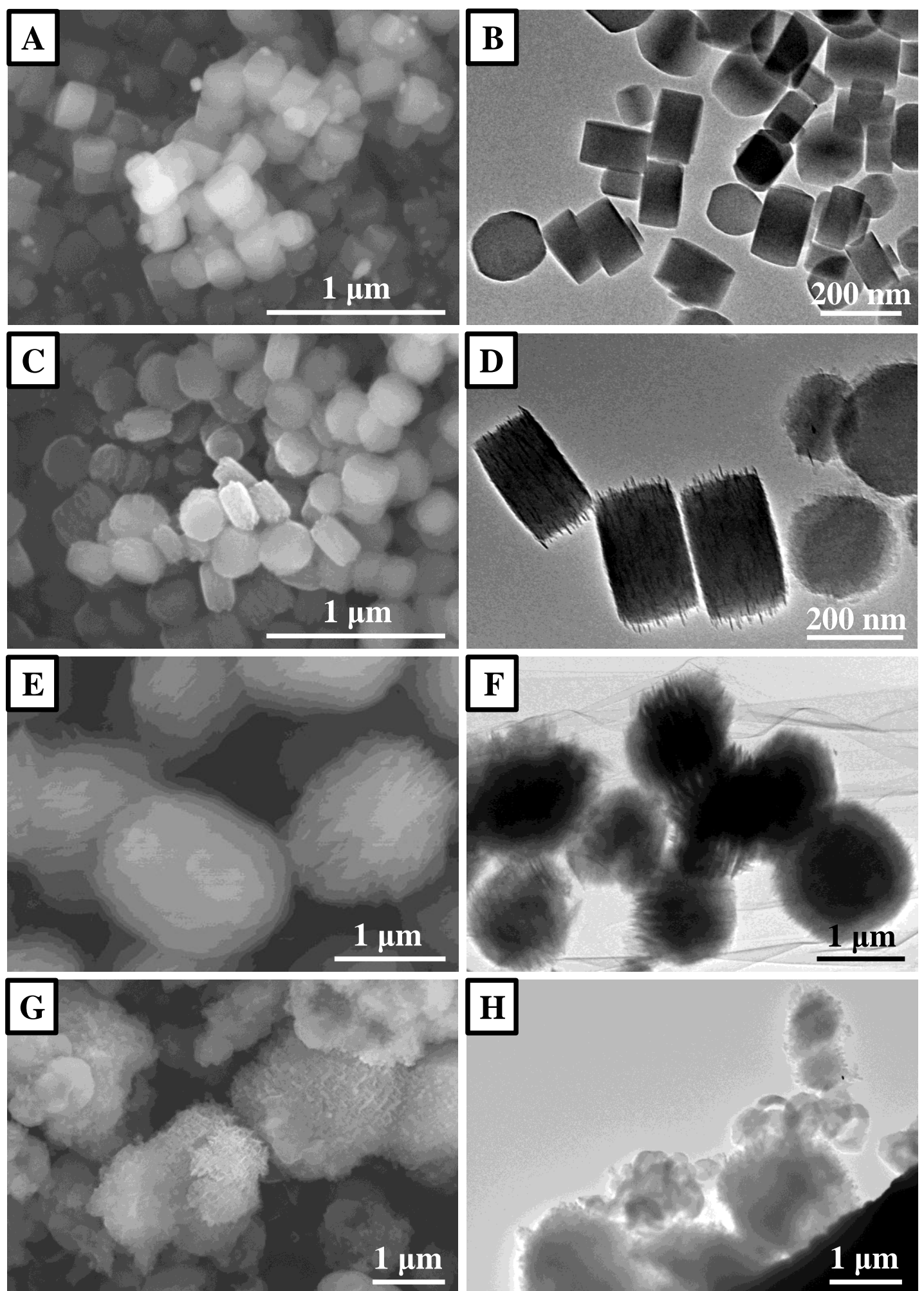

Figure 2. SEM and TEM images of HLBM zeolite with $\mathrm{Si} / \mathrm{Al}=300$ after 5 days of hydrothermal synthesis. (SEM: (A) HLBM-0/36, (C) HLBM-1/36, (E) HLBM-3/36, and (G) HLBM-5/36. TEM: (B) HLBM-0/36, (D) HLBM-1/36, (F) HLBM-3/36, and (H) HLBM-5/36.) 

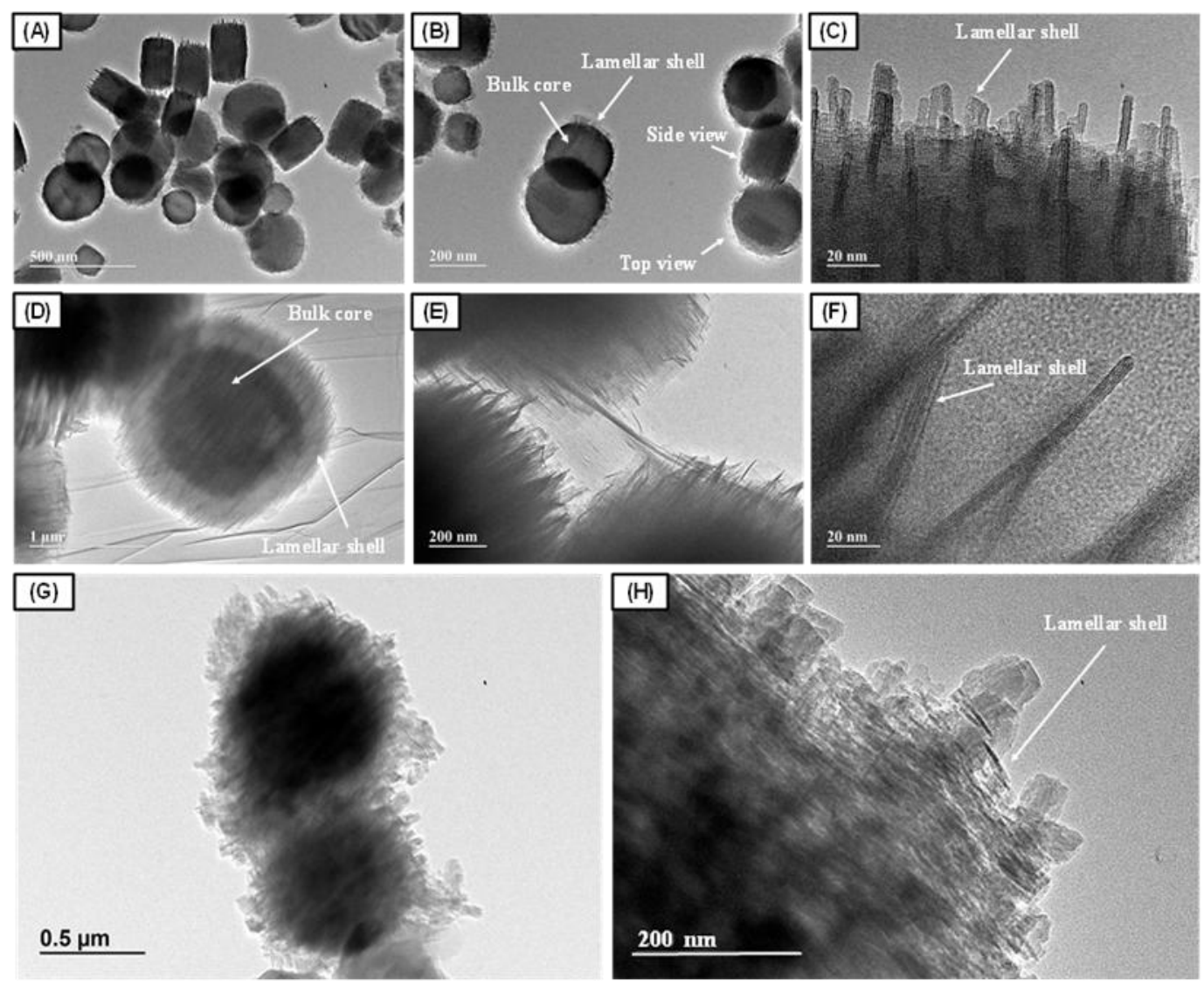

Figure 3. High resolution TEM images of HLBM zeolite with $\mathrm{Si} / \mathrm{Al}=300$ after 5 days of hydrothermal synthesis. ((A)-(C): HLBM-1/36; (D)-(F): HLBM-3/36, and (G)-(H): HLBM-5/36, respectively.) 

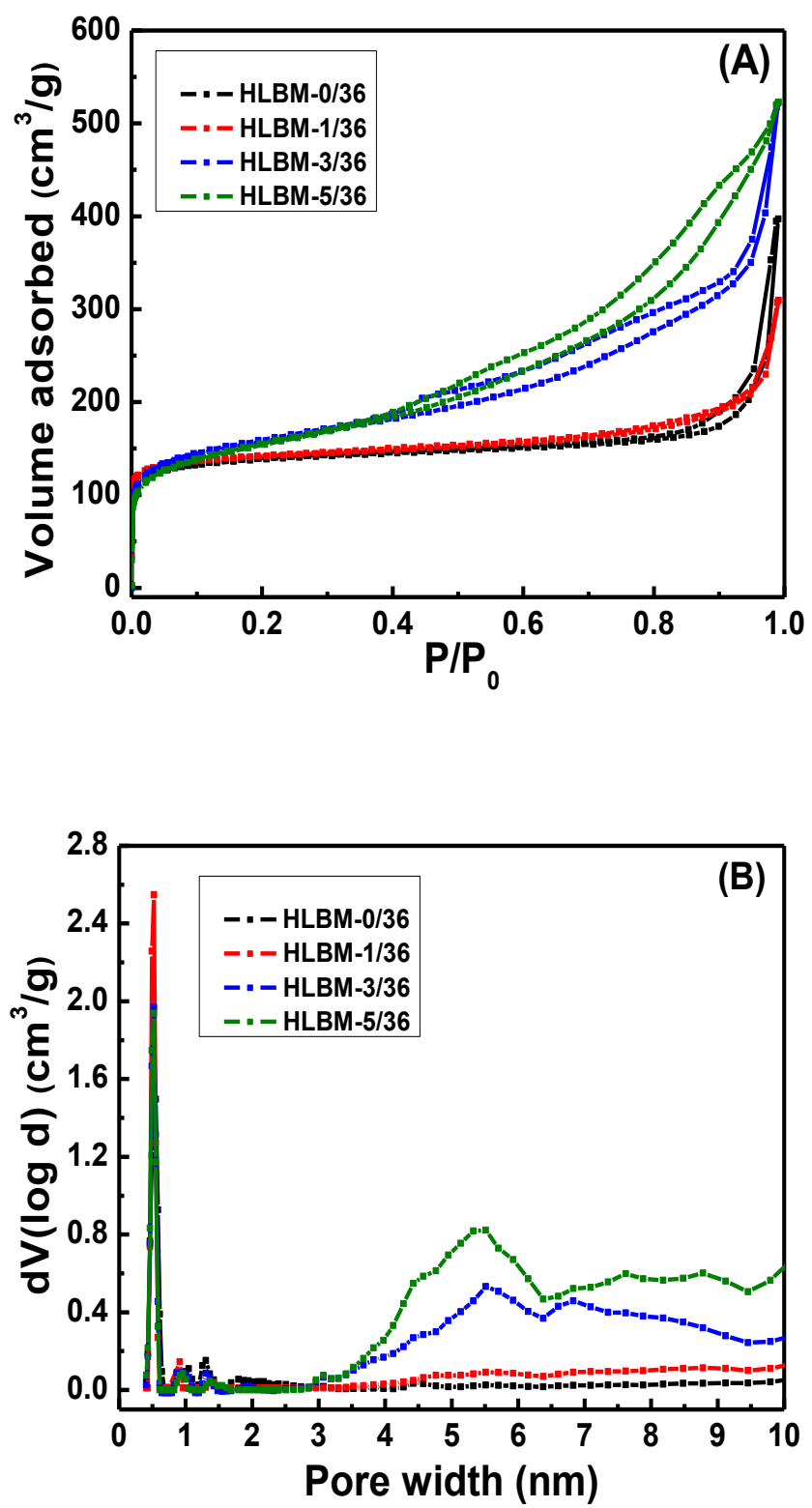

Figure 4. Ar adsorption-desorption isotherms (A) and the corresponding NLDFT pore size distributions (B) derived from Ar adsorption branch of the HLBM zeolite catalysts. 

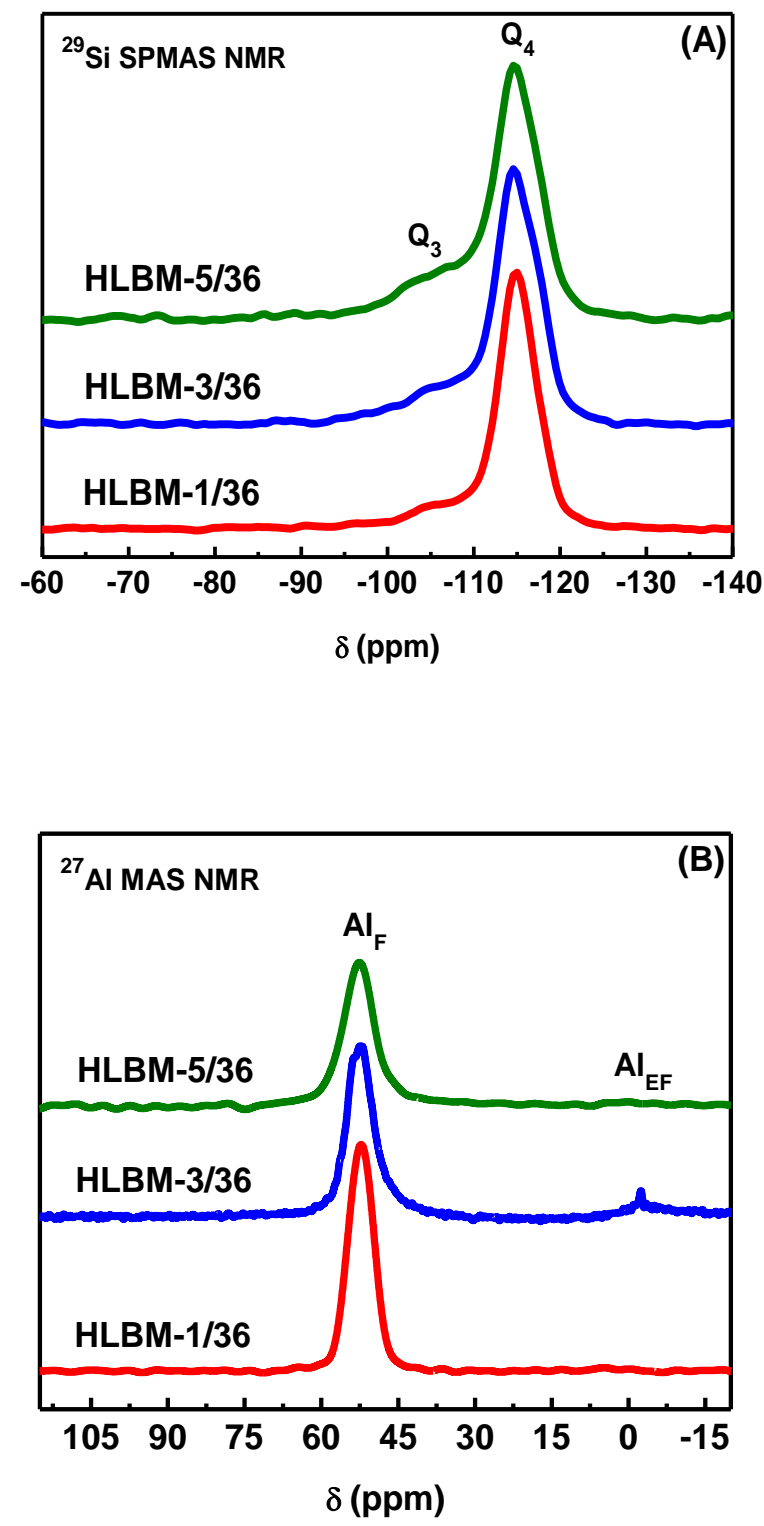

Figure 5. Solid state ${ }^{29}$ Si SP MAS NMR (A) and ${ }^{27}$ Al MAS NMR (B) spectra of HLBM zeolite catalysts. 

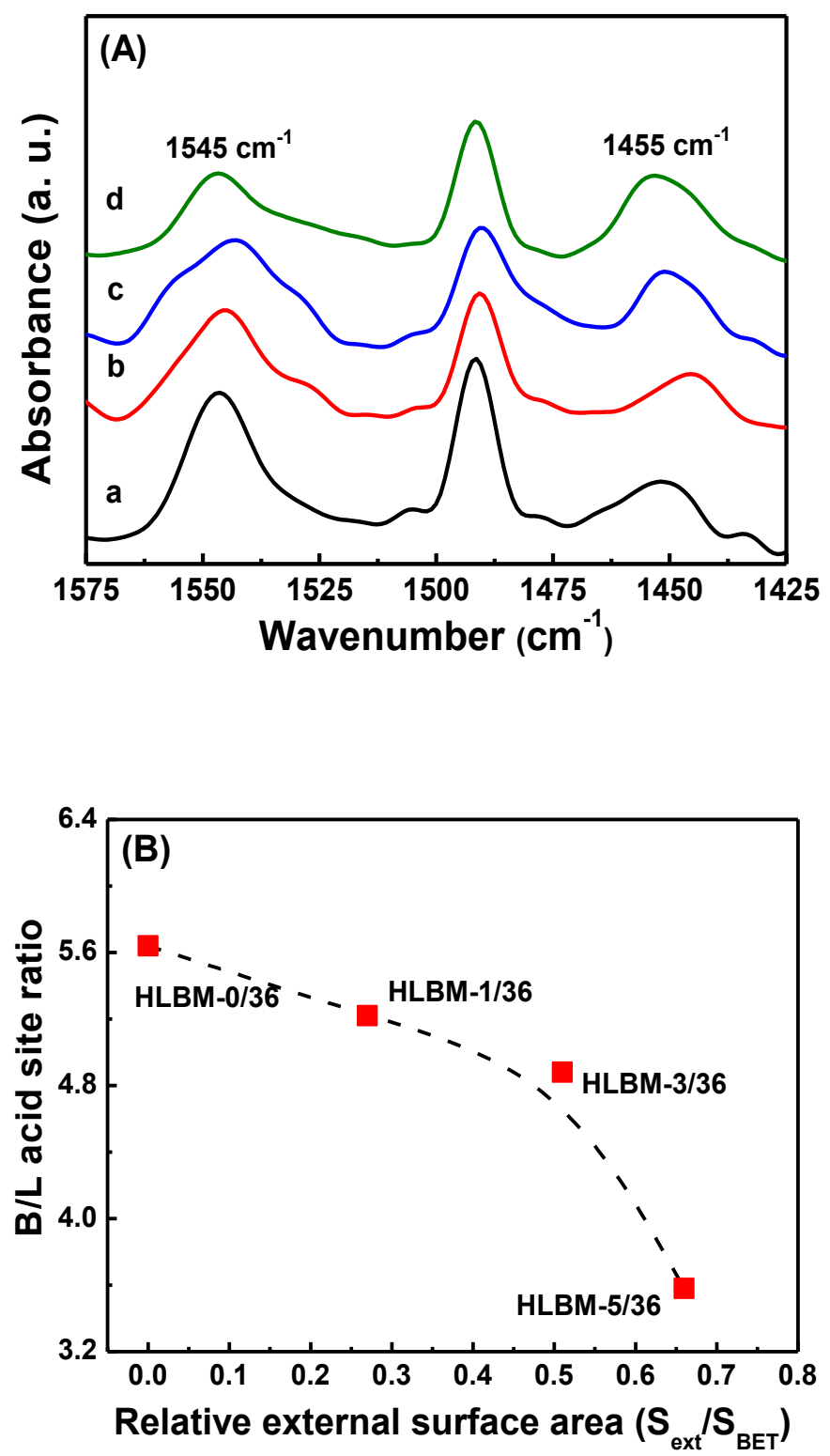

Figure 6. (A) FTIR spectra of adsorbed pyridine ((a) HLBM-0/36, (b) HLBM-1/36, (c) HLBM3/36, and (d) HLBM-5/36, respectively) on HLBM zeolite catalysts and (B) B/L acid site ratio versus relative external surface area of HLBM zeolite catalysts. 

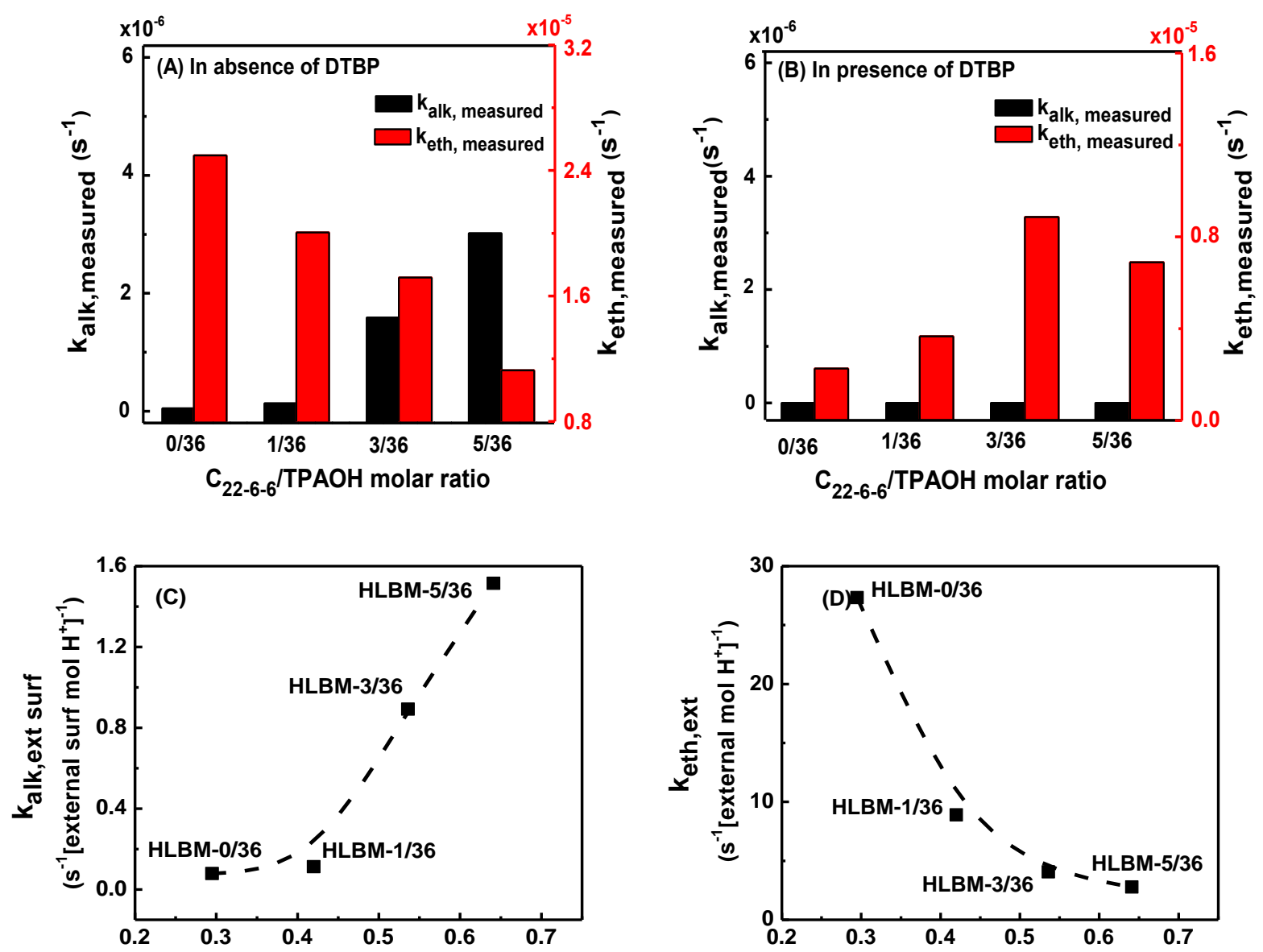

Relative external surface area $\left(\mathrm{S}_{\mathrm{ext}} / \mathrm{S}_{\mathrm{BET}}\right)$

Relative external surface area $\left(\mathrm{S}_{\mathrm{ext}} / \mathrm{S}_{\mathrm{BET}}\right)$

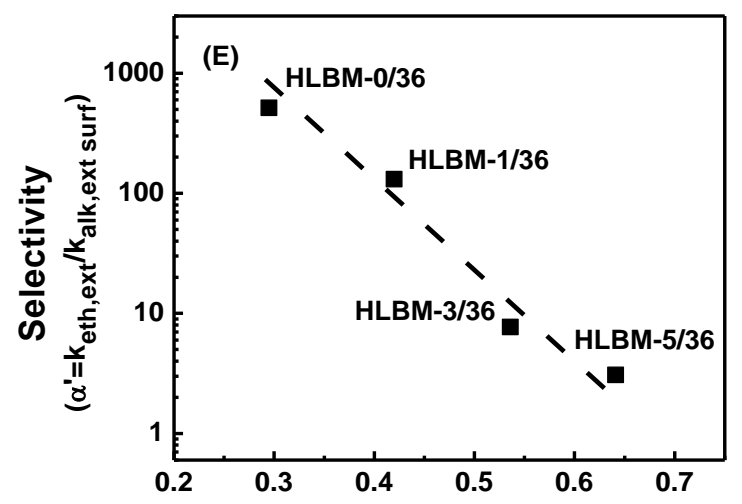

Relative external surface area $\left(\mathrm{S}_{\mathrm{ext}} / \mathrm{S}_{\mathrm{BET}}\right)$

Figure 7. Measured alkylation and etherification rate constants of benzyl alcohol with mesitylene reaction in absence of DTBP (A) and in presence of DTBP (B), rate constants of alkylation on external surface acid sites (C) and etherification on external acid sites (D), and selectivity (E) versus relative external surface area of HLBM zeolite catalysts. 

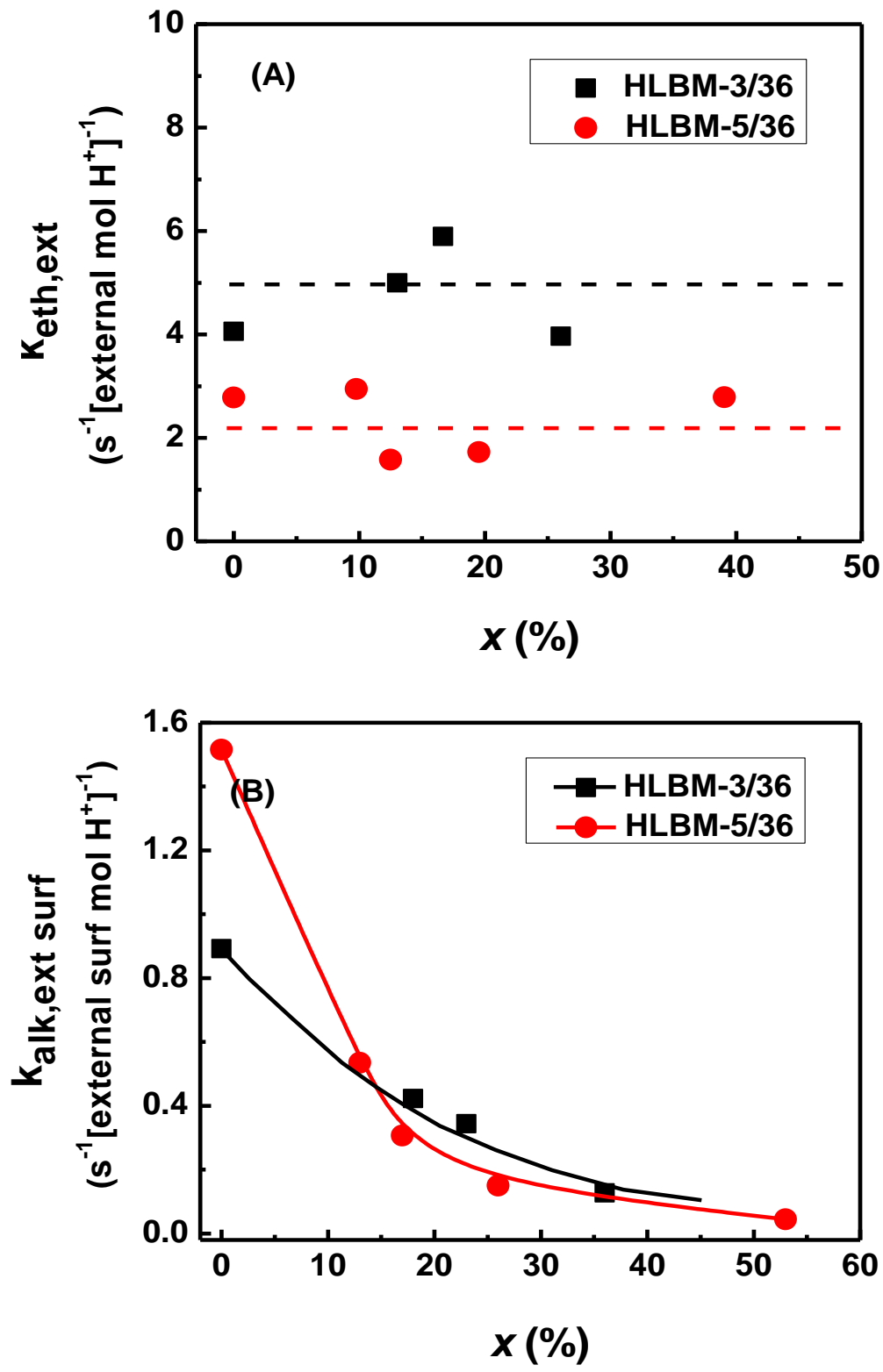

Figure 8. Rate constants of etherification on external acid sites (A) and alkylation on external surface acid sites (B) of HLBM-3/36 and HLBM-5/36, respectively, with accumulative addition of DTBP to poison the external acid sites. $(x(\%)$ is the percentage of active external acid sites (A) and the percentage of active external surface acid sites (B) that were poisoned by DTBP.) 
Graphic Abstract

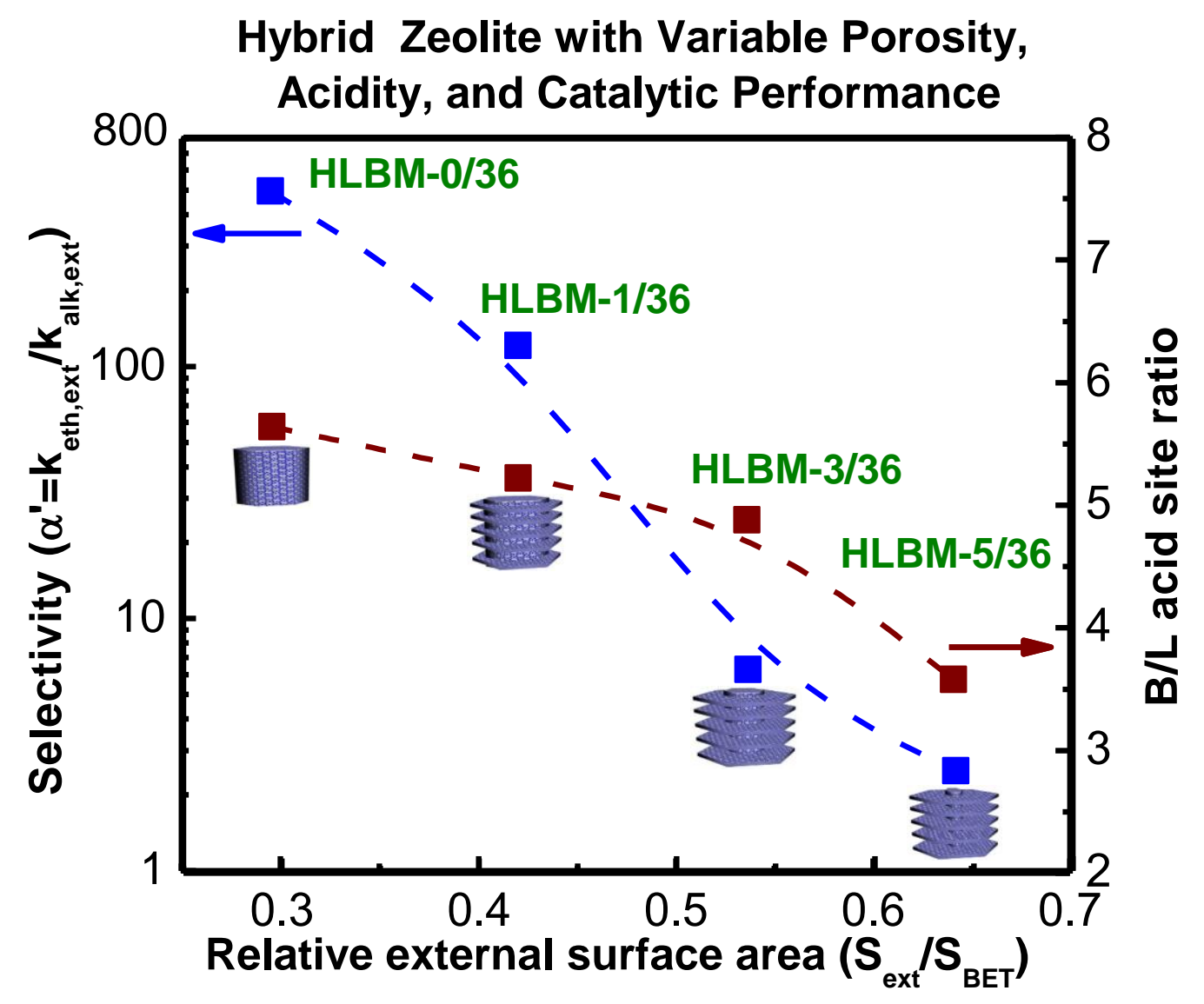

